\title{
Development of Autonomous Actions to Enable the Next Decade of Ocean World Exploration
}

\author{
Corresponding Author: Glenn E. Reeves, \\ Jet Propulsion Laboratory (JPL), \\ California Institute of Technology, \\ glenn.e.reeves@jpl.nasa.gov
}

Co-Authors: Brett A. Kennedy (JPL), Grace H. Tan-Wang (JPL), Paul G. Backes (JPL), Steve A. Chien (JPL), Vandi Verma (JPL), Kevin P. Hand (JPL), Cynthia B. Phillips (JPL)

This work was carried out at the Jet Propulsion Laboratory, California Institute of Technology, under a contract with the National Aeronautics and Space Administration (80NM0018D0004).
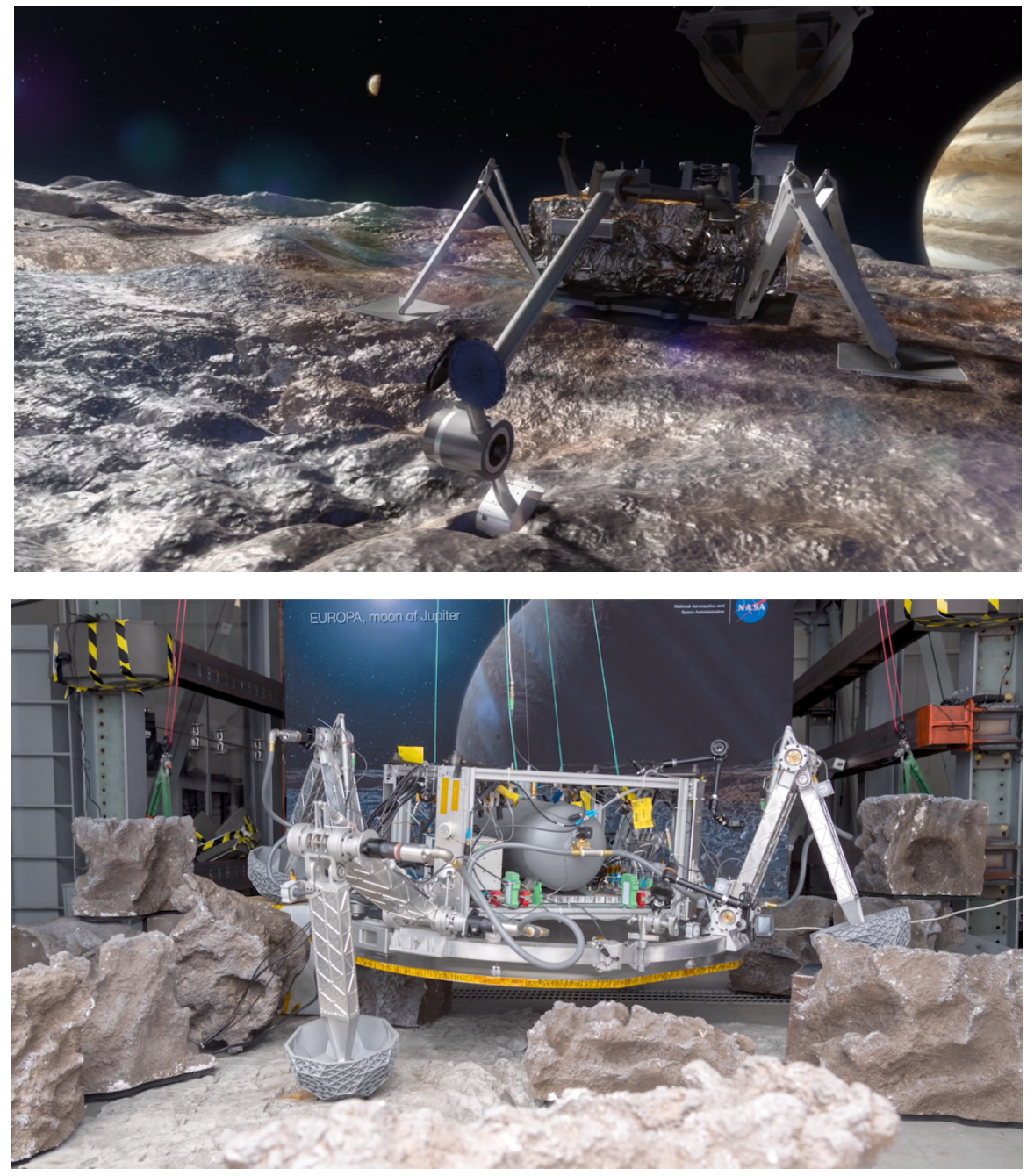

(C) 2020. California Institute of Technology. Government sponsorship acknowledged

Pre-Decisional Information - For Planning and Discussion Purposes Only CL\#20-3666 


\section{Executive Summary}

The development of autonomous actions aboard surface systems is of great value to the exploration of ocean worlds, and the in situ exploration of much of our solar system. The ability of landers to operate with more autonomy than current landers and rovers will enable more and better science, with an overall reduction in cost for comparable missions. In this paper we identify the array of challenges that confront in situ exploration on an ocean world and how many of these challenges are, and have been, addressed (and in some cases resolved) in the early development work for NASA's Europa Lander mission concept. We identify the pathway for constructing a highly autonomous surface system that enables ocean worlds exploration across an array of targets. Our key developments include:

- A system-wide architecture effort to identify the mission activities, and the onboard decisions, that will be required for time- and resource-efficient missions. This activity identifies the onboard autonomous behaviors and the necessary supporting architecture, such as the sensing required. A holistic approach to autonomy overall is the intent, including identifying computation needs, strategies for the judicious use of energy, applicability of multiple methods for sample acquisition, fault and failure reaction strategies, learning options from surface interactions, and self-calibration and assessment techniques.

- A focused investigation of the autonomy related to sample acquisition, including mechanism and end-effector tool development, the development of representative algorithms for workspace assessment, sample/excavation target identification, dynamic tool use/control, and sensing needed to ascertain acquisition success. This investigation also includes adaptation based on surface interactions.

- The construction of both a hardware-in-the-loop (HITL) testbed and a software-plus-simulation ("SoftSim") testbed environment to enable the implementation of multiple "prototype" "systems". The testbed environments will have both the fidelity and flexibility to represent the trade space for the most challenging tasks related to autonomous functionality.

- The exploration of onboard versus ground operator responsibility for mission activities. The uncertainties of the surface environment, and the influence of operators/scientists in the execution of the specific activities, deserves consideration and experimentation. The proper mix will vary with the mission target, but all are likely to need to balance the amount of science and engineering data for a given uplink or downlink opportunity to maintain a compelling operational tempo. (Europa allows a relatively low direct-to-Earth communications link for about $50 \%$ of a 86 hour europan day.)

- An exploration of autonomy enabling architectural and design choices for the surface spacecraft. A surface mission with significant autonomy goals will require expanded resiliency by design, spanning from sensing and perception to alternative strategies to accomplish the required mission activities. Less visible, but still critical to autonomous behaviors, will be sufficient computation, sufficient memory and storage, and effective fault/interruption detection. Designing a system with fewer resource limitations may be the right trade to reduce the overall complexity of implementation.

(C) 2020. California Institute of Technology. Government sponsorship acknowledged

Pre-Decisional Information - For Planning and Discussion Purposes Only CL\#20-3666 
- An exploration of how to achieve the continuation of activities even in the presence of interruptions of functionality or failures due to the environment.

\section{Introduction}

The robotic exploration of our solar system can be significantly enhanced through the development and implementation of autonomy for many aspects and phases of mission operations. Autonomy can be used to increase the operational efficiency of a mission, helping to conserve engineering resources while simultaneously increasing the science return of a mission. From sampling on the surfaces of Venus and Ocean Worlds, to acquiring data during the flyby of a Kuiper belt object, many science goals and mission targets will benefit from developments and investments in autonomy. On Venus, the harsh temperature and pressure of the surface impose a limit on the lifetime of a landed vehicle, making autonomy an attractive answer to acquiring and processing samples. On Ocean Worlds such as Europa and Enceladus, planetary protection concerns and mission cost and complexity could make radioisotope power sources less attractive, leading to some shorter lived missions that operate with primary batteries. Such missions would also benefit significantly from elements of autonomous operations. Finally, efforts to deploy low-cost missions that fly-by, orbit, or even land on worlds throughout our Solar System will be enabled by the ability to autonomously achieve targeted science and execute ranked onboard operations.

Here we highlight developments in autonomy that have been made possible as part of the 2016-2020 Europa Lander Mission Concept. We also detail areas for future development and investment. Though our description focuses on the specific case of the Europa Lander mission concept, these advancements can be applied to many other ocean worlds and planetary missions.

\section{What is 'autonomy'?}

Here we define autonomy as the ability of a vehicle to achieve the mission goals while operating independently of external control. At the same time, the vehicle must perceive and react when presented by the uncertainties of interacting with Europa (or any other world). Autonomy must include both the ability to self-direct, and the self-sufficiency needed to operate independently. The overall system that achieves these characteristics must be designed from conception to support autonomy.

\section{Autonomy and the Europa Lander Mission Concept}

We refer the reader to the Europa Lander Study 2016 Report for background information on the Europa Lander science and the mission concept.

The baseline Europa Lander mission concept employs onboard autonomy to efficiently collect and analyze samples, and then transmit prioritized information to Earth $^{\text {ii }}$. Figure 1 shows some examples of specific systems under development for surface operations. Importantly, operational efficiency and urgency is only one of a number of challenges that an in situ mission at Europa, or any ocean world, would be required to address. The use of autonomy is an obvious architectural solution to many of the challenges, but our 
ability to truly capitalize on its promise requires an encompassing examination of the challenges and required capabilities of the eventual system. This is not a simple reapplication of prior techniques, nor is it sufficient to assume that there is direct relationship to strategies used in other in situ missions. Although a spacecraft that must operate on the surface of Europa faces similar challenges that spacecraft on Mars may have addressed, Europa isn't Mars, and there are unique challenges that amplify the critical need for onboard autonomous capabilities. The Europa Lander pre-project has assessed those challenges and is in the process of building the strategies, techniques, framework, and tools to apply to the development ${ }^{\mathrm{iii}}$.

\section{Pre-Project Advanced Development for Autonomy}

The Europa Lander pre-project is a mature concept that has been evolving since the Mission Concept Review ${ }^{1}$ with project funds for advanced development work in autonomy and other areas. This effort is multifaceted as it is intended to advance the maturity of the specific technology, architecture, and design challenges. The Europa Lander pre-Project is funded to investigate the breadth and scope of the autonomous capabilities potentially needed to ensure a successful surface mission phase.

\section{Motivations and Solutions for Autonomous Operations}

- Surface Uncertainty: The spacecraft would land on the surface of Europa with surface knowledge at a scale larger than what will be needed for sample acquisition (meters versus centimeter). Since the surface topography and the specific material properties of the surface material would have a significant understanding uncertainty prior to landing, the spacecraft must be designed for a range of possible scenarios, and the system must be able to autonomously adapt as required.

○ Advanced Developments and Future Work: First, this uncertainty requires the onboard system to be perceptive enough to refine the measurements sufficiently in order to identify the proper action. Second, there must be a diversity in the methods and mechanisms such that the proper action is available. Since sample acquisition is a critical function, the advanced development tasks in this theme address multiple methods for performing the surface sample acquisition requirements (excavation to $>10 \mathrm{~cm}$, collection, processing, and delivery of the sample material to the instruments). The construction of both a hardware-in-the-loop (HITL) testbed and a software-plussimulation ("SoftSim") testbed environment enable the implementation of multiple "prototype" "systems" (see cover image). The HITL testbed will be used to compare and contrast different end-effector/tool choices with manipulators that vary from low to high dexterity (3 to $6+$ degrees of freedom). The HITL testbed will have representative control and kinematics allowing for the development of dynamic and adaptive control strategies for excavation, collection, and tool exchange. The SoftSim environment enables the investigation and experimentation of the other behaviors of the surface vehicle including planner/executive/learning agents, energy conservation, fault/failure/interruption recovery, and instrument data assessment. The testbed environments will have both the fidelity and flexibility to represent the trade space for most challenging tasks related to autonomous functionality.

\footnotetext{
1 The Europa Lander Mission Concept Review was successfully completed in November 2018.

(C) 2020. California Institute of Technology. Government sponsorship acknowledged Pre-Decisional Information - For Planning and Discussion Purposes Only CL\#20-3666
} 


\section{Ambient Testbeds}

- Variety of ambient testbeds to investigate: autonomy algorithms, effects of system compliance, initial tool investigations $300+$ ambient tests to date. Most significant testbeds shown below. Variety of low DOF testbeds as well.
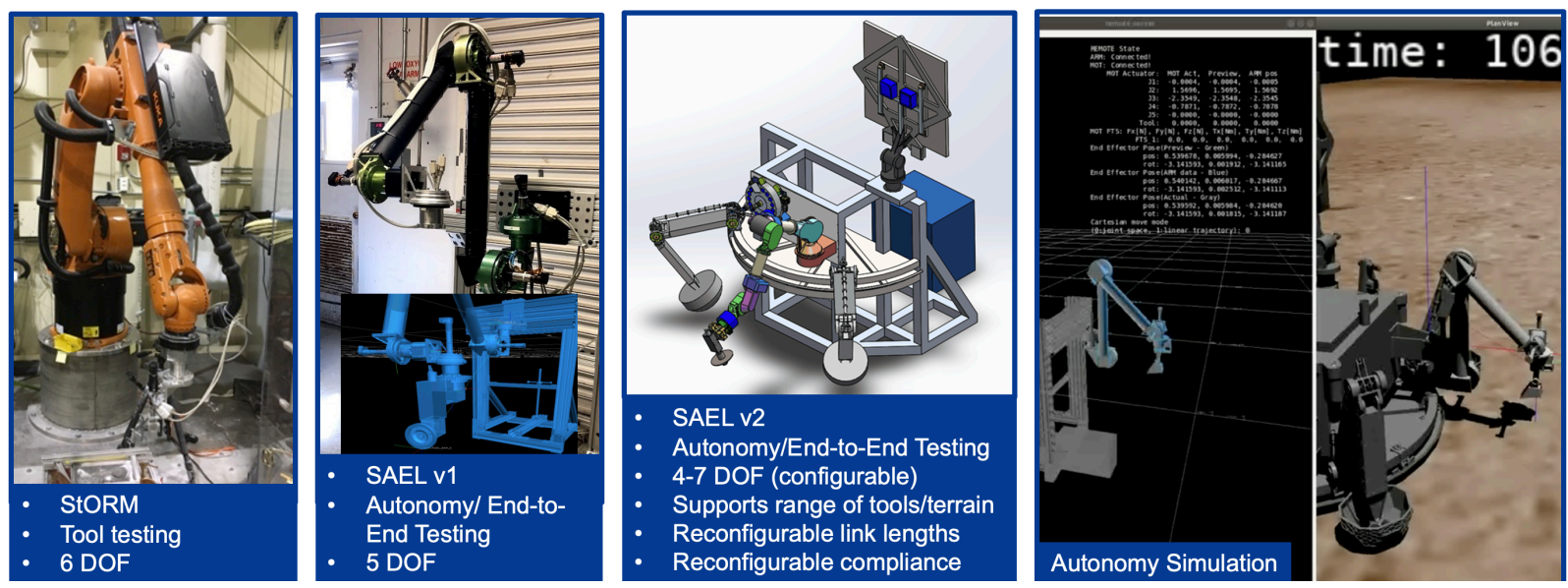

Figure 1. Autonomy developments for the Europa Lander mission concept have included a variety of hardware and software testbeds that enable close coupling across multiple subsystems. Shown above are several examples of testbeds for sample acquisition and delivery that have, and will continue to be used for end-to-end testing.

\section{- Europa's Distance and Orbit constrain the timing and frequency of} communication with Earth: Europa will be approximately 4 AU from Earth during the surface mission phase. At this distance, data rates are significantly less than Mars missions enjoy, and the light time introduces additional latency. Furthermore, Europa is tidally locked to Jupiter with an orbital period of $\sim 86$ hours. This means that Earth is only visible to the Lander for $\sim 40$ hours per orbit. Without autonomy, these constraints would lead to significant idle time on the surface of Europa, during which energy for heating and spacecraft maintenance would be consumed, without yielding any science return. The advanced development in this area explores the trades and constraints for adapting to or mitigating these constraints, and conducting science for longer periods.

- Advanced Developments and Future Work: When the Earth is in view, the questions of when to expend the energy to be ready to receive, when to transmit, and for how long to transmit are imposed design complexities emergent from the limited energy resources. For example, any urgency due to Ground-in-the-Loop (GITL) involvement in operations introduces a desire for "spontaneous" downlink to convey to ground operators the data they need to make any decisions affecting mission activities. A similar challenge is knowing when to turn on the radio to receive communication/direction from Earth, since it is undesirable to leave the receiver on continuously. The periods right after Earthrise and just before Earthset are also likely anchor points for communication, as they border the exit and entry where DTE/DFE ${ }^{2}$ communication is possible. On the related note - and illustrative of the challenges involved - the baseline mission has the vehicle landing on the Europa surface with $\sim 10$ hours before Earthset ${ }^{3}$. This limited time convolves many prerequisite activities with

\footnotetext{
${ }^{2}$ Direct-to-Earth/Direct-from-Earth

${ }^{3}$ The time limited duration isn't easy to expand as the landing time is significantly constrained by landing system requirements as well as the constraints trajectory design that enable reasonable $\Delta \mathrm{V}$ requirements. (c) 2020. California Institute of Technology. Government sponsorship acknowledged Pre-Decisional Information - For Planning and Discussion Purposes Only CL\#20-3666
} 
communicating a successful landing, communicating the state of the vehicle, and potentially providing some mission science return before the first Earth set. All of these need to be accomplished autonomously onboard for efficiency.

- Fault/Fail, Recover, Continue: In order to keep up a reasonable operational tempo, the system should not be dependent on GITL intervention to recover from interruptions, faults, or failures. For instance, the system needs to detect, recover, and continue the mission without ground intervention if faced with a radiation-induced single event effect (SEE) that manifests as an interruption in the electronics.

- Advanced Developments and Future Work: Developments in this area include the identification of detection methodologies that expose SEE effects, understanding the necessary state/information needed to permit recovery, and identifying the techniques and behaviors necessary to orchestrate recovery.

- Balancing Autonomous Operation and GITL: The balance of onboard autonomy and a GITL decision process is not only based upon the design of the flight segment, but also on the sensitivities and concerns of the human stakeholders. While a fully autonomous vehicle could be constructed and could execute without GITL, the concept for operations (and far more likely scenario) is that there will be a carefully orchestrated coordination of flight and ground behaviors and responsibilities to allow for surface operations to change once the vehicle is on the surface.

- Advanced Developments and Future Work: Exploring where the possible boundaries for autonomous flight vehicle responsibility versus ground operations team responsibility is part of the trade and exploration space. The advanced development work has included the examination of the flight versus ground responsibilities and the decision processes required by human operators. By systematically evaluating the information required in order to make such decisions, the migration of such decisions from ground to the spacecraft can be assessed. The assessment includes the viability of any required sensing, constructing the information set, assessing the complexity of the algorithmic decision logic, and assessing the presumed variations in the resulting onboard behavior. All of these are scrutinized with a focus on being able to demonstrate, test, and certify/trust the autonomous behavior.

- Energy Storage and Management: For the delta-MCR baseline design of the Europa Lander, the energy density of the primary batteries, plus the mass of the batteries ${ }^{4}$ themselves, yield a predicted lifetime to 30 to $\sim 60$ Earth days with traditional technologies. The hardware design and onboard software would need to autonomously modify energy consumption rates as a function of the mission activities. In addition, the system would need to predict energy/lifetime in order to maintain sufficient capacity in order to return science/instrument data before expiring.

- Advanced Developments and Future Work: Maximizing the surface lifetime is critical to lowering overall mission risk. Early development work includes battery life/capacity testing and battery chemistry tuning with industry partners (e.g.,

\footnotetext{
${ }^{4}$ Battery mass and structure mass are the two largest contributors to the overall mass of the surface vehicle. The ratio of "landed" mass to launch mass is a factor of 6 . 
Eagle Picher). In addition, the exploration of lower power electronics is an ongoing activity. The end goal is a system that autonomously adjusts energy consumption as a function of activities.

- Information Flow to Earth: The baseline mission uses a Direct-to-Earth, Directfrom-Earth communication architecture with a highly efficient High-Gain Antenna design, a high-power radio/amplifier, and an arrayed set of Deep Space Network (DSN) antennas. The effective downlink rate is expected to be $\sim 48 \mathrm{kbps}$. This bandwidth is a significant driver on battery capacity (and thus mission life) as it couples the radio/amplifier on-time with the quantity of data transmitted. Ideally the spacecraft uses its last modicum of energy while it is transmitting science data. It is conceivable that the system may switch to exclusively transmitting data if the already collected high value data reaches a threshold where further activities become of low predicted utility.

- Advanced Developments and Future Work: Increasing the total volume and the information density of the data that must be returned requires a combination of approaches. For example, JPL has developed and patented ${ }^{\text {iv }}$ a High-Gain antenna design that has unprecedented performance. This is a critical step toward increasing the information return capability. Complementary to this are the strategies and techniques to increase the value of the information that is transmitted. This includes the traditional strategies of decimation and compression, as well as investigating onboard instrument data assessment. It is possible that machine learning techniques can be applied to allow the onboard system to order communications activity based on an autonomous assessment of instrument data and resulting priority of the measurements.

- Building Hardware to Enable Autonomy: Autonomy cannot be viewed as an addition to the system; it must be considered and incorporated in the early architecture and design of the overall vehicle.

○ Advanced Developments and Future Work: A surface mission with significant autonomy goals will require expanded resiliency by design. Most notably, perception methods, both in the form of robust sensing and the algorithms needed to interpret the sensed scene/situation, deserve investigation. Almost equally important are literal and functionally overlapping methods to achieve the critical mission objectives - sample acquisition, sample transport, sample processing, and sample analysis. Less visible, but still critical to autonomous behaviors, will be sufficient computation, sufficient memory and storage, and effective fault/interruption detection.

- Synergetic Instrument Design: Historically, instruments have been treated as "payload' on the spacecraft to be operated and managed in coordination with the "spacecraft", but mostly loosely coupled with regard to both design and operations. Of course, there are specific points of design coupling like interface definitions, power requirements, and mechanical interfaces that are precise and impact the overall system. There are also coupled onboard operations, like sample handoff, that are drivers on interoperability, but these two areas envelope the scale of the onboard inter-design complexity for e.g., Mars surface missions. Similarly, vehicle and instrument operations teams intentionally operate semi-independently to the level reasonable for planning and 
coordinate at the highest level reasonable to check for known conflicts and over use of resources, as this provides greater flexibility and autonomy for the diverse and significant ground team to operate. Moving forward with autonomy will foster a closer coupling between the payload system and the spacecraft system, ensuring that one system 'knows' what the other is doing and how best to prioritize actions and manage resources.

○ Advanced Developments and Future Work: The Europa Lander mission concept employs a close coupling of instrument-to-spacecraft, and vice-versa, as necessary for an optimized usage of resources, as well as better able to deal with uncertainty autonomously. Understanding how instruments and their operation can be accommodated is one of the thematic purposes of the ICEE- $2^{\mathrm{v}}$ instrument development investments. The development in this area is intended to identify the requirements on instruments and the surface vehicle that enable an autonomous system that is designed to operate as coordinated whole. In many cases, the instruments themselves would need to provide the autonomous behaviors that traditionally might have required a GITL cycle to effect operations. This includes not just the instruments, but also the sample acquisition and sample delivery mechanisms, both of which have received funding through the advanced development work.

\section{Summary}

Autonomy can enable increased science return per dollar spent on a mission. In the case of the Europa Lander mission concept, autonomy is an important component of enabling a lower-cost, lower-risk (especially as relates to planetary protection), nonradioisotope power system mission.

The autonomy design space spans hardware, algorithms, functions, ground operations, and mission activities in an end-to-end manner. The challenges of autonomy are numerous and require early attention, exploration, trade studies, and experimentation to determine the right baseline for the eventual mission. This development is already in progress with the Europa Lander pre-project development activities. The mission concept is mature and passed its deltaMission Concept Review in November of 2018. If allowed to move into Phase-A, this mission concept could help pioneer new technologies, techniques, and operations that are applicable to the exploration of many bodies throughout the Solar System. This mission concept provides the civilization-scale science that is of interest to much of humanity, and it presents technological challenges - such as autonomy - that are the hallmark of NASA's charter to innovate and inspire.

\footnotetext{
i Science Definition Team Report: https://europa.nasa.gov/resources/58/europa-lander-study-2016-report/. In addition, a presentation and slides on the mission concept can be found here: https://www.europainsitu.caltech.edu/

ii Please see Decadal paper first authored by Ray Crum, "Advanced Technology Developments for Europa Lander and other In-Situ Ocean World Missions."

iii Autonomy Challenges and Development for Europa Lander, JPL Limited Release Record LRR050823

iv HGA Patent: https://patents.google.com/patent/US10680345B2/en?inventor=Nacer+E. + Chahat

v ICEE-2 overviews: https://www.lpi.usra.edu/opag/meetings/apr2019/presentations/Schulte.pdf and https://www.jpl.nasa.gov/missions/web/absscicon/03-ICEE2-Overview-at-AbSciCon-2019$\underline{\text { Krajewski.pdf }}$ 